\title{
Phillygenin attenuates LPS-induced acute lung injury of newborn mice in infantile pneumonia
}

\author{
Meiyan Wang ${ }^{1}$, Qian Cai ${ }^{2, *}$
}

\author{
${ }^{1}$ Department of Pediatrics, The First \\ Affiliated Hospital of Medical College of \\ Shihezi University, 832000 Shihezi City, \\ Xinjiang Uygur Autonomous Region, \\ China \\ ${ }^{2}$ Department of Pediatrics, The Third \\ Xiangya Hospital of Central South \\ University, 410013 Changsha, Hunan \\ Province, China

\section{*Correspondence} \\ caiqian_054@163.com \\ (Qian Cai)
}

\begin{abstract}
Purpose: The aim of this study was to assess the effect of phillygenin (PHI) on lipopolysaccharide (LPS)-induced acute lung injury (ALI) and understand its underlying mechanisms.

Methods: Mice were separated into four different groups at random, including LPS, LPS + PHI $(5 \mathrm{mg} / \mathrm{kg})$, LPS + PHI $(50 \mathrm{mg} / \mathrm{kg})$ and control group. The two LPS + PHI groups were intraperitoneally administered with PHI after LPS intratracheal administered for $1 \mathrm{~h}$. Subsequently, the lung tissues of different groups were collected and evaluated by $\mathrm{H} \& \mathrm{E}$ staining and W/D (W/D) ratio. The inflammatory cytokines in BALF or lung tissue were also assessed. Western blot assay was applied to examine the expressions of TLR4, MyD88, and NF- $\kappa \mathrm{B}$.

Results: The ameliorated pathological changes and lung W/D ratio demonstrated that PHI dramatically suppressed the lung injury levels. PHI strikingly reduced the number of inflammatory cell counts and total protein concentration in BALF. In addition, PHI attenuated expression of IL- $1 \beta$ and TNF- $\alpha$ in BALF and lung tissue. Furthermore, it was confirmed that PHI alleviated LPS-induced ALI via TLR4/MyD88/NF- $\kappa$ B pathway. Conclusions: Together the above results show that PHI attenuates LPS-induced ALI via inactivation of TLR4/MyD88/NF- $\kappa$ B pathway in newborn mice.
\end{abstract}

\section{Keywords}

Phillygenin; Acute lung injury; TLR4; MyD88; NF- $\kappa \mathrm{B}$

\section{Introduction}

Pediatric pneumonia is a typical disease in infants and a common cause of infant death [1]. ALI caused by infection is a serious disease that requires intensive care. Recent epidemiological studies indicated that ALI is a major global health problem and it remains a considerable challenge for clinicians [2-4]. Pathological manifestations of ALI include impaired alveolar epithelial integrity, increased permeability, and inflammatory activation, leading to pulmonary edema, hypoxemia, atelectasis, hyaline membrane, and even respiratory failure [5]. However, the effective treatment of ALI still has some limitations due to its complex pathogenesis. Thus, it is important to delve into the pathogenesis of ALI and seek new treatment strategies to cure the ALI.

Toll-like receptors (TLRs) lead innate immune receptors to recognize pathogen-related molecular patterns, not only initiating primary responses to invasive pathogens but also inducing adaptive immune responses [6]. TLR4 induces inflammatory responses by recognizing endogenous ligands associated with tissue damage [7]. Myeloid differentiation primary response 88 (MyD88), as a shared adaptor molecule, usually causes activation of several inflammatory cytokine genes and NF- $\kappa \mathrm{B}$ [8]. Previous studies have indicated that the TLR4/MyD88 is pivotal to LPS-induced ALI, it can be reduced through suppressing the TLR4/MyD88/NF- $\kappa \mathrm{B}$ axis [9]. Consequently, regulating TLR4/MyD88/NF- $\kappa \mathrm{B}$ is possibly an effective way to treat ALI.

Phillygenin (PHI), a metabolite in the fruit of forsythia suspensa, is present in the rapeseed family of Forsythia [10]. Pharmacological experiments have proved that PHI plays an anti-inflammatory activity by suppressing the production of nitric oxide (NO) and the nuclear factor NF- $\kappa \mathrm{B}$ [11]. PHI can also stimulate the anti-inflammatory function of macrophages by inducing the release of IL-10 and TGF- $\beta$, besides reducing the IL- $1 \beta$ and TNF- $\alpha$ expression levels [12]. LPS-induced LX2 cell inflammation is inhibited by suppressing TLR4/MyD88/NF- $\kappa \mathrm{B}$ expression [13]. However, the role of PHI in LPS-induced ALI remains elusive.

In this study, PHI was shown to reduce LPS-induced ALI in neonatal mice, regulate LPS-induced lung injury scores, reduce inflammatory factor expression levels, and block the TLR4/MyD88/NF- $\kappa$ B activity. Thus, PHI could be a potential strategy for the treatment of LPS-induced ALI. 


\section{Materials and methods}

\subsection{Animals and treatment protocols}

3-8 day old C57 mice were purchased from BIORAY LABORATORIES Inc. (Shanghai, China). All experimental procedures and animal welfare were carried out in accordance with the National Institutes of Health Laboratory Animal Care and Use Guidelines [14], the protocols used were approved by the Ethical Committee of the First Affiliated Hospital of Medical College of Shihezi University (Approval No. MCSU2019061).

\subsection{Establishment of ALI model}

Mice were separated into 4 different groups $(\mathrm{n}=6)$ at random, including LPS, LPS + PHI $(5 \mathrm{mg} / \mathrm{kg}$; Aladdin, Shanghai, China), LPS + PHI (50 mg/kg) and control group. PHI (5 or $50 \mathrm{mg} / \mathrm{kg})$ was intraperitoneally injected after LPS $(5 \mathrm{mg} / \mathrm{kg}$; Sigma-Aldrich) intratracheal injection for $1 \mathrm{~h} \mathrm{[15].}$

\subsection{Histopathological analysis of lung tissues}

For evaluating the lung injury, the right lung were removed after challenge with LPS for $24 \mathrm{~h}$, then the right lung were embedded in paraffin after fixation in $4 \%(\mathrm{v} / \mathrm{v})$ paraformaldehyde. Next, the right lung tissues were cut into about 3.5 $\mu \mathrm{m}$ sections and stained with hematoxylin and eosin solution (Sigma-Aldrich). Lastly, the pathological lung tissues of mice were observed under optical microscope (Olympus, Japan).

\subsection{Measurement of lung W/D ratio}

After LPS challenge for $24 \mathrm{~h}$, the left lung were then excised and the wet weight was obtained. Then, the left lung of mice were incubated in $60{ }^{\circ} \mathrm{C}$ oven for 3 days to obtain the dry weight [16].

\subsection{Bronchoalveolar lavage fluid (BALF) collection}

BALF was collected for the analysis of cytokine production. Briefly, the BALF was collected by intratracheal intubation. The lungs were rinsed for 3 times with $0.6 \mathrm{~mL}$ of ice-cold PBS and a total of approximately $1.4 \mathrm{~mL}$ of BALF was collected. Finally, the BALF was centrifuged at $1600 \mathrm{rpm}$ for $9 \mathrm{~min}$, and the supernatant was obtained and stored at $-80{ }^{\circ} \mathrm{C}$ [17].

\subsection{Cell counts and protein concentration measurements in BALF}

Macrophages and neutrophils in the BALF were counted by the Diff-Quik ${ }^{\mathrm{TM}}$ staining system (International Reagents Corp., Japan). Then the cell counts were performed by Olympus microscope at $40 \times$ magnification. For protein concentration measurements, the supernatants in BALF were used to measure the total protein concentration through BCA Protein Assay Kit (CoWin Biotechnology).

\subsection{ELISA}

For detecting the IL-1 $\beta$ (ab197742; Abcam, Cambridge, MA, USA) and TNF- $\alpha$ (Cat ab208348; Abcam) protein levels, ELISA kits were used. The expression of TNF- $\alpha$ and IL- $1 \beta$ in BALF or cells were assayed with corresponding ELISA kits. All measurements were performed in triplicate independently.

\section{$2.8 \mathrm{qPCR}$}

Trizol reagent (Invitrogen, Grand Island, NY, USA) was applied to extract the total RNA from collected cells. The quantity and integrity of extracted total RNA were evaluated on a Nano Drop 1000 spectrophotometer (Thermo Fisher Scientific, Inc.). The expression of IL- $1 \beta$ and TNF- $\alpha$ were detected through qRT-PCR by the SYBR Premix EX Taq (Takara, Japan). $\beta$-actin were used as the endogenous reference genes to normalize mRNA expression levels. The relative expression of IL- $1 \beta$ and TNF- $\alpha$ in each experimental group were analyzed by the $2^{-\triangle \triangle \mathrm{Ct}}$ method. All reactions were performed in triplicates. Primer sequences are shown in Table 1.

\subsection{Western blot}

Briefly, cells were washed in precooled PBS buffer 3 times, and the total protein was extracted by RIPA buffer (Beyotime). Protein concentration was measured by using BCA protein assay kits (CoWin Biotechnology). Equal amount of total proteins were loaded and separated with SDS-PAGE. Then, the proteins were transferred to polyvinylidene difluoride membranes (PVDF; Millipore) and blocked by $5 \%$ non-fat milk at room temperature for $1 \mathrm{~h}$. Proteins were recognized by incubation with specific primary antibodies: TLR4 (ab217274, $1:$ 1,500; Abcam), MyD88 (ab2064, $1: 1,500$; Abcam), p-NF- $\kappa$ B p65 (ab86299, 1 : 1,500; Abcam), NF- $\kappa$ B p65 (ab16502, 1 : 1,500; Abcam), and $\beta$-actin (Rabbit Antibeta Actin antibody, ab8227, $1: 1,500$; Abcam) overnight at $4{ }^{\circ} \mathrm{C}$. Then, the membranes were further incubated with horseradish peroxidase (HRP)-conjugated goat anti-rabbit immunoglobulin G secondary antibody (ab205718, 1 : 1,500; Abcam) and the bands on the membranes were visualized by the ECL chemiluminescence reagent (Beyotime). The $\beta$-actin was used to normalize the analyzed samples and protein bands were quantified by gray value analysis using ImageJ software (National Institutes of Health).

\subsection{Statistical analysis}

All data are shown as the mean \pm SD from three independent experiments. Comparisons between two groups were performed using one-way ANOVA test. $p$ values of $<0.01$ (twotailed) were considered to indicate a statistically significant difference.

\section{Results}

\subsection{Increased PHI attenuated LPS-induced histopathologic changes and inhibited LPS-induced increases of W/D ratio in mice}

It is well known that LPS administration in mice has been established as an in vivo animal model of ALI [18]. In this 
TA B L E 1. Primers for TNF- $\alpha$ and IL-1 $\beta$ and reference genes.

\begin{tabular}{lll} 
Gene & Primer & Sequence $\left(5^{\prime} \longrightarrow 3^{\prime}\right)$ \\
\multirow{2}{*}{ TNF- $\alpha$} & Forward & GCCACCACGCTCTTCTGTCTAC \\
& Reverse & GGGTCTGGGCCATAGAACTGAT \\
IL-1 $\beta$ & Forward & ACCTTCCAGGATGAGGACATGA \\
& Reverse & CTAATGGGAACGTCACACACCA \\
\multirow{2}{*}{$\beta$-actin } & Forward & GTGACGTTGACATCCGTAAAGA \\
& Reverse & GCCGGACTCATCGTACTCC \\
\hline
\end{tabular}

A

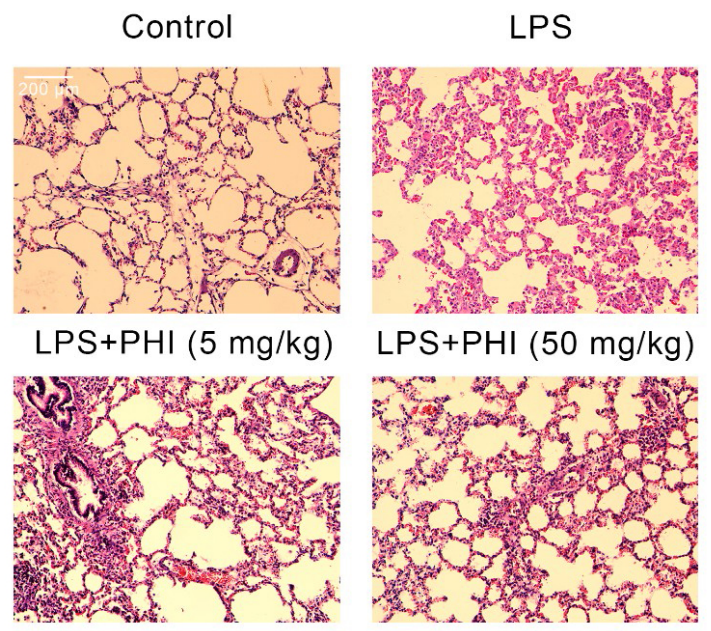

B

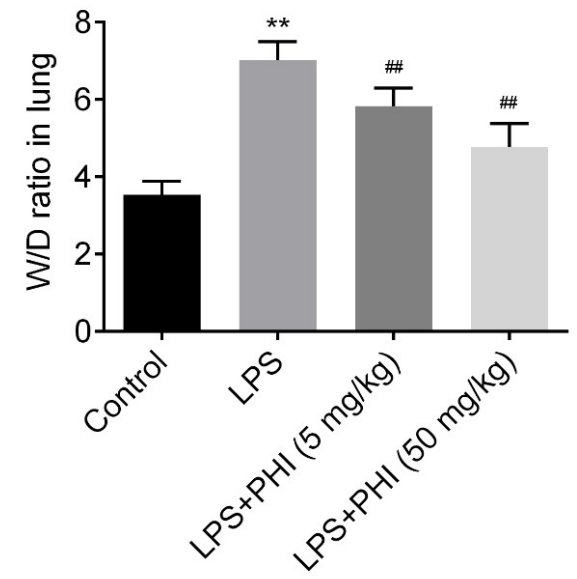

F I G U R E 1. PHI ameliorated LPS-induced pathological changes in lung tissue of ALI mice. (A) Hematoxylin and eosin staining in each group, including control group, LPS group, LPS + PHI $(5 \mathrm{mg} / \mathrm{kg})$ group, and LPS + PHI (50 mg/kg) group (magnification: $\times 200$ ). (B) Effects of PHI $(5$ and $50 \mathrm{mg} / \mathrm{kg}$ ) on the lung W/D ratio of LPS-induced ALI mice. $* * p<0.01$ versus control group and ${ }^{\# \#} p<0.01$ versus LPS alone group.

research, the animal ALI disease model was first established. The lung tissues of control group mice did not show any pathological changes (Fig. 1A). On the other hand, LPS challenge group showed apparent histopathologic changes with extensive alveolar wall thickness caused by pulmonary edema and marked alveolus collapse. These histopathological changes were ameliorated by the administration of PHI in different doses. It was clearly observed that the high dose of PHI (50 $\mathrm{mg} / \mathrm{kg}$ ) had a more significant improvement than the low dose group $(5 \mathrm{mg} / \mathrm{kg})$. Later, the lung W/D ratio was assessed to demonstrate the edema (Fig. 1B). The results revealed that W/D ratio was markedly higher in the LPS group after $24 \mathrm{~h} \mathrm{LPS}$ challenge by comparing to the control group. However, administration of PHI dose-dependently suppressed LPS-induced increase in lung W/D ratio, particularly at the concentration of $50 \mathrm{mg} / \mathrm{kg}$.

\subsection{Increased PHI reduced the number of inflammatory cells counts and total protein concentration in BALF of LPS-induced ALI mice}

Since vascular leakage is a marker of ALI, the total protein concentration and inflammatory cell number in BALF should be determined. The total protein concentration (Fig. 2A) and total inflammatory cells number (Fig. 2B) were first assessed. By comparing to the control group, the mice with LPS challenge had significantly increased protein concentration and total inflammatory cells number, while mice treated with PHI dose-dependently suppressed the LPS-induced increase in protein concentration and total inflammatory cell number, particularly at the concentration of $50 \mathrm{mg} / \mathrm{kg}$. Furthermore, the number of inflammatory cells after LPS treatment for $24 \mathrm{~h}$ was also analyzed to evaluate the value of PHI on LPS-induced lung inflammation. The numbers of neutrophils (Fig. 2C) and macrophage (Fig. 2D) were markedly enhanced in LPS challenge group compared to control group. PHI suppressed the increase of neutrophils and macrophage, and high dose of PHI had a more significant effect on neutrophils and macrophage.

\subsection{Increased PHI ameliorated inflammatory response in BALF and lung tissue of LPS-induced ALI mice}

The levels of pro-inflammatory cytokines (TNF- $\alpha$ and IL-1 $\beta$ ) in BALF and lung tissue were measured to validate the potential anti-inflammatory value of PHI in LPS-induced ALI mice. It showed that LPS dramatically upregulated the expression 
A

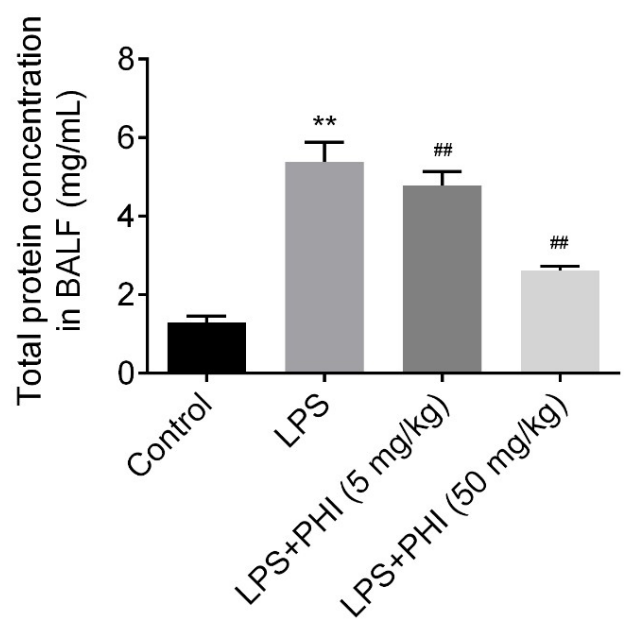

C

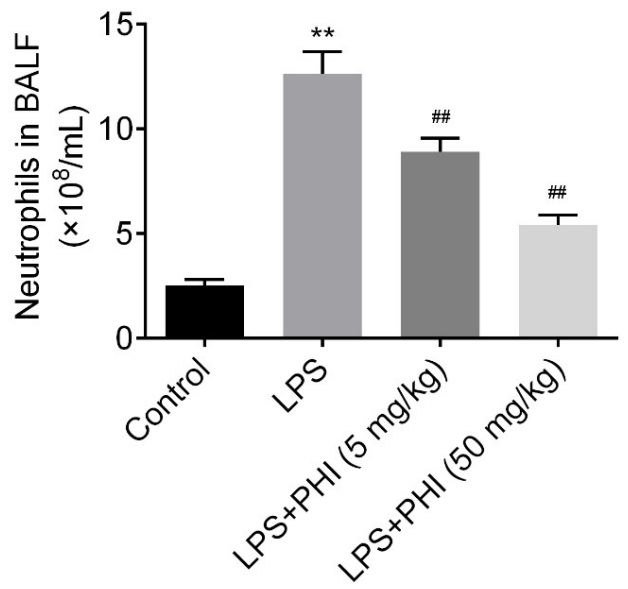

B

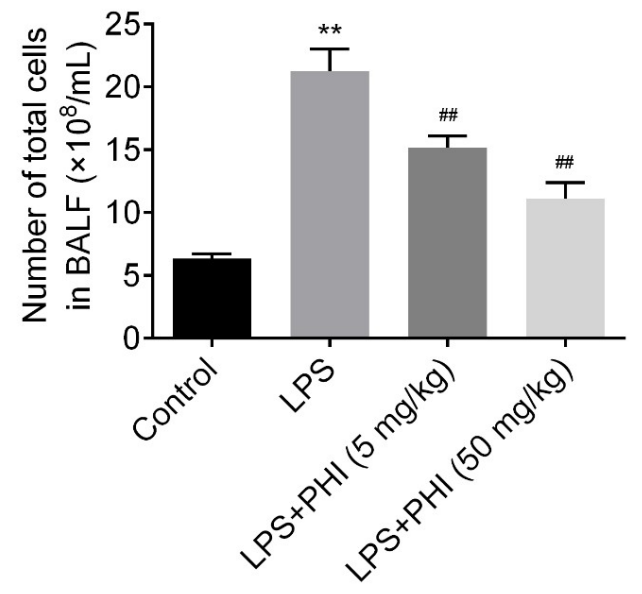

D

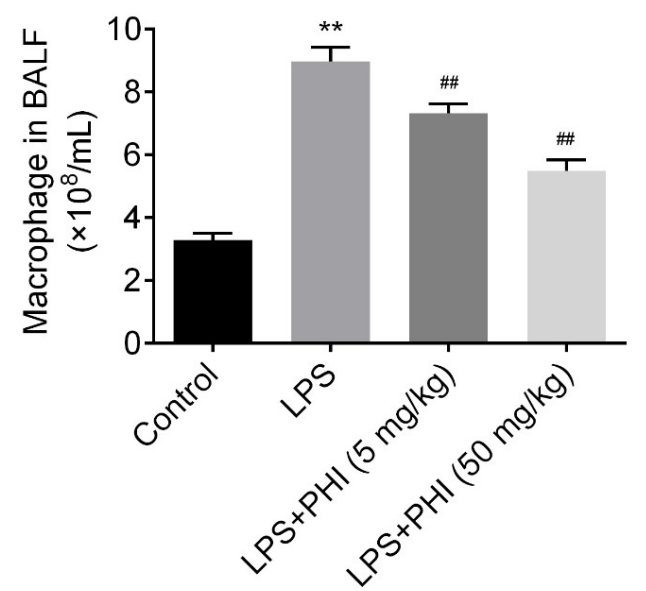

F I G U R E 2. PHI reduced LPS-induced vascular leakage and inflammatory cells infiltration in lung tissue of ALI mice. (A) Total protein concentration in BALF. (B) Number of total cells in BALF. (C) Number of neutrophil in BALF. (D) Number of macrophages in BALF. ${ }^{* *} p<0.01$ versus control group and ${ }^{\# \#} p<0.01$ versus LPS alone group.

of pro-inflammatory cytokines in both BALF (Fig. 3A,B) and lung tissue (Fig. 3C,D) of LPS-induced ALI mice. However, increased PHI administration, particularly at the concentration of $50 \mathrm{mg} / \mathrm{kg}$, strikingly attenuated the expression levels of pro-inflammatory cytokines induced by LPS. These results indicated that PHI treatment has a therapeutic efficacy by attenuating inflammatory response in both BALF and lung tissue of LPS-induced ALI mice, and a high dose of PHI had a more marked therapeutic efficacy in both BALF and lung tissue of LPS-induced ALI mice.

\subsection{Increased PHI alleviated LPS-induced ALI by modulating TLR4/MyD88/NF- $\kappa$ B pathway}

To test the hypothesis that PHI attenuated LPS-induced inflammation by modulating TLR4/MyD88/NF- $\kappa$ B pathway, qPCR assay (Fig. 4A) and western blot assay (Fig. 4B) were applied to measure the expression levels of TLR4, MyD88, and NF- $\kappa \mathrm{B}$ in lung tissues at $24 \mathrm{~h}$ post LPS treatment. The phosphorylation level of NF- $\kappa \mathrm{B}$ protein showed a dramatic upregulation in LPS group after $24 \mathrm{~h}$ challenge with LPS. However, the treat- ment of PHI dose-dependently reversed LPS-induced ALI, especially at the concentration of $50 \mathrm{mg} / \mathrm{kg}$, indicating that PHI could suppress NF- $\kappa$ B signaling pathway. Moreover, the up-regulated protein expressions of TLR4 and MyD88 could also be suppressed by administration of PHI ( 5 or 50 $\mathrm{mg} / \mathrm{kg}$ ). And a high dose of PHI had a more significant effect on suppressing protein expressions of TLR4 and MyD88 induced by LPS challenge. These data indicated that administration of PHI attenuated the LPS-induced ALI by modulating TLR4/MyD88/NF- $\kappa$ B pathway.

\section{Discussion}

This study confirmed that PHI protected against LPS-induced ALI in mice, and its anti-inflammation properties could partly explain this effect. Specifically, the results indicated that PHI ameliorated LPS-induced ALI and exerted an antiinflammatory effect on LPS-induced ALI via inhibiting of TLR4/MyD88/NF- $\kappa$ B pathway. Based on this finding, PHI may work as a potential therapeutic target for ALI therapy. 
A

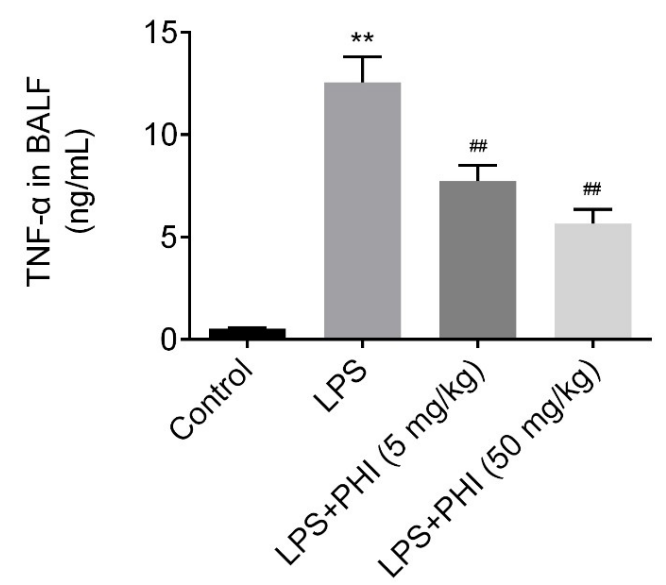

C

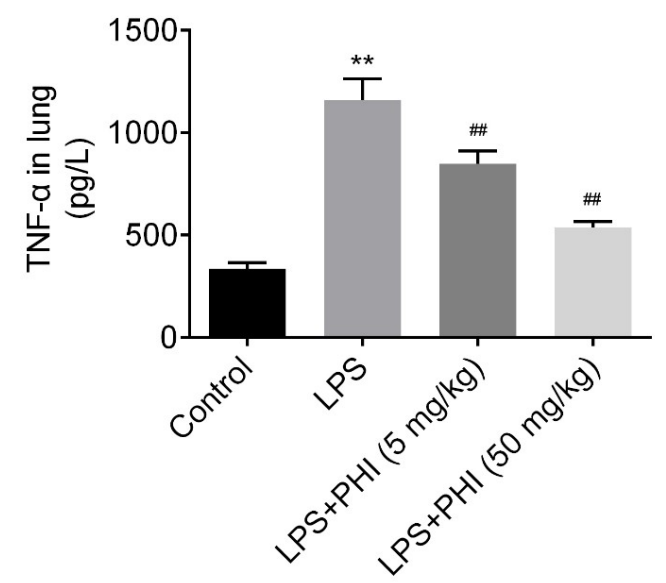

B

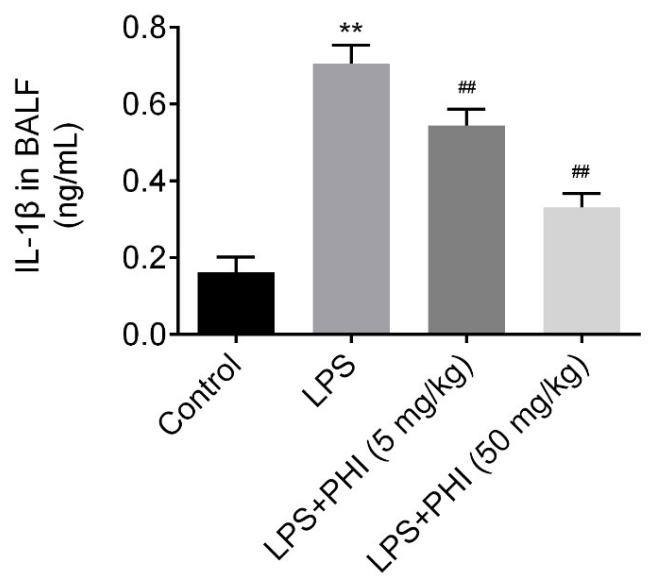

D

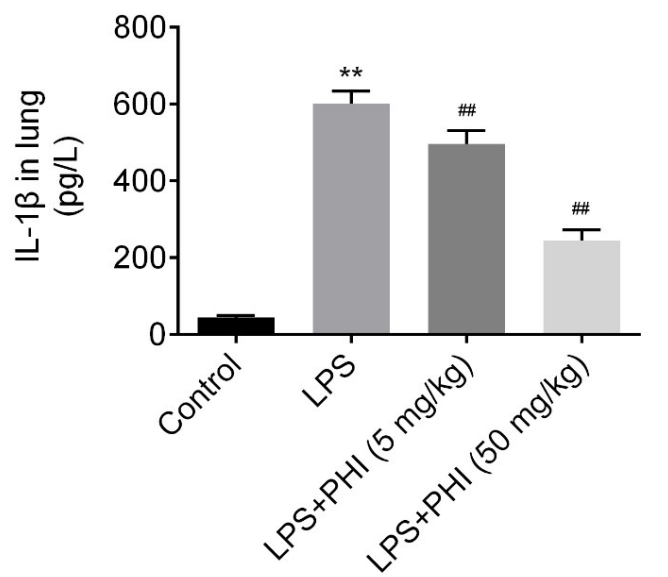

FIGURE 3. PHI reduced LPS-induced inflammatory response in BALF and lung tissue of ALI mice. (A) TNF- $\alpha$ expression levels in BALF. (B) IL-1 $\beta$ expression levels in BALF. (C) TNF- $\alpha$ expression levels in lung tissue. (D) IL-1 $\beta$ expression levels in lung tissue. ${ }^{* *} p<0.01$ versus control group and ${ }^{\# \#} p<0.01$ versus LPS alone group.

ALI is an acute lung inflammation that disturbs barriers between epithelium and endothelium [3]. It is one of the syndromes with high mortality due to various causes inside the lung. Although many promising pharmacological therapies have been reported, none of them are effective in reducing the mortality. Thus, it is urgent and necessary to explore an effective method to treat ALI. The well-known animal model of ALI mice is usually established by LPS treatment [19]. In the present study, LPS-induced ALI mice were first constructed as animal model through LPS challenge for subsequent studies.

$\mathrm{PHI}$ is a major bioactive component of the fruits of forsythia suspensa and it possesses various pharmacological properties such as anti-inflammatory activities [20]. Several other studies have reported that PHI markedly showed a protective effect against LPS-induced cytotoxicity [21]. Due to multi-targeted actions of PHI, it could have a broad prospect in the development of new and safe cytoprotective drugs as a promising natural product [22]. However, few research had concentrated on the pharmacological value of PHI in LPS-induced ALI and the potential mechanism of it has not been reported yet.

Growing number of researches have confirmed that inflam- matory response has a crucial role in induction of LPS-induced ALI [23-25]. Pro-inflammatory cytokines increase lung epithelial permeability, which in turn leads to accumulation of neutrophils and lung tissue injury, resulting in pulmonary edema [26]. Pro-inflammatory cytokines increasing in the BALF and lung tissues of patients are used as one of the typical biomarkers in monitoring the initiation and maintenance of LPS-induced ALI [27]. Interestingly, accumulating evidence indicates that PHI is associated with inflammatory response [28], which prompted us to test if PHI also alleviates LPSinduced ALI by improving the inflammatory mediator production. This study indicated that PHI significantly diminished total protein concentration and total inflammatory cells number in BALF. Besides, we also showed that PHI effectively reduced macrophages and neutrophils in the BALF. Furthermore, the expression of inflammatory cytokines in BALF and lung tissues were also dramatically lower than those in the control group. These results demonstrated that the amelioration of LPS-induced ALI by PHI is related to its inflammation inhibitory properties.

TLR4 is a well-known primary innate immune receptor for 
A
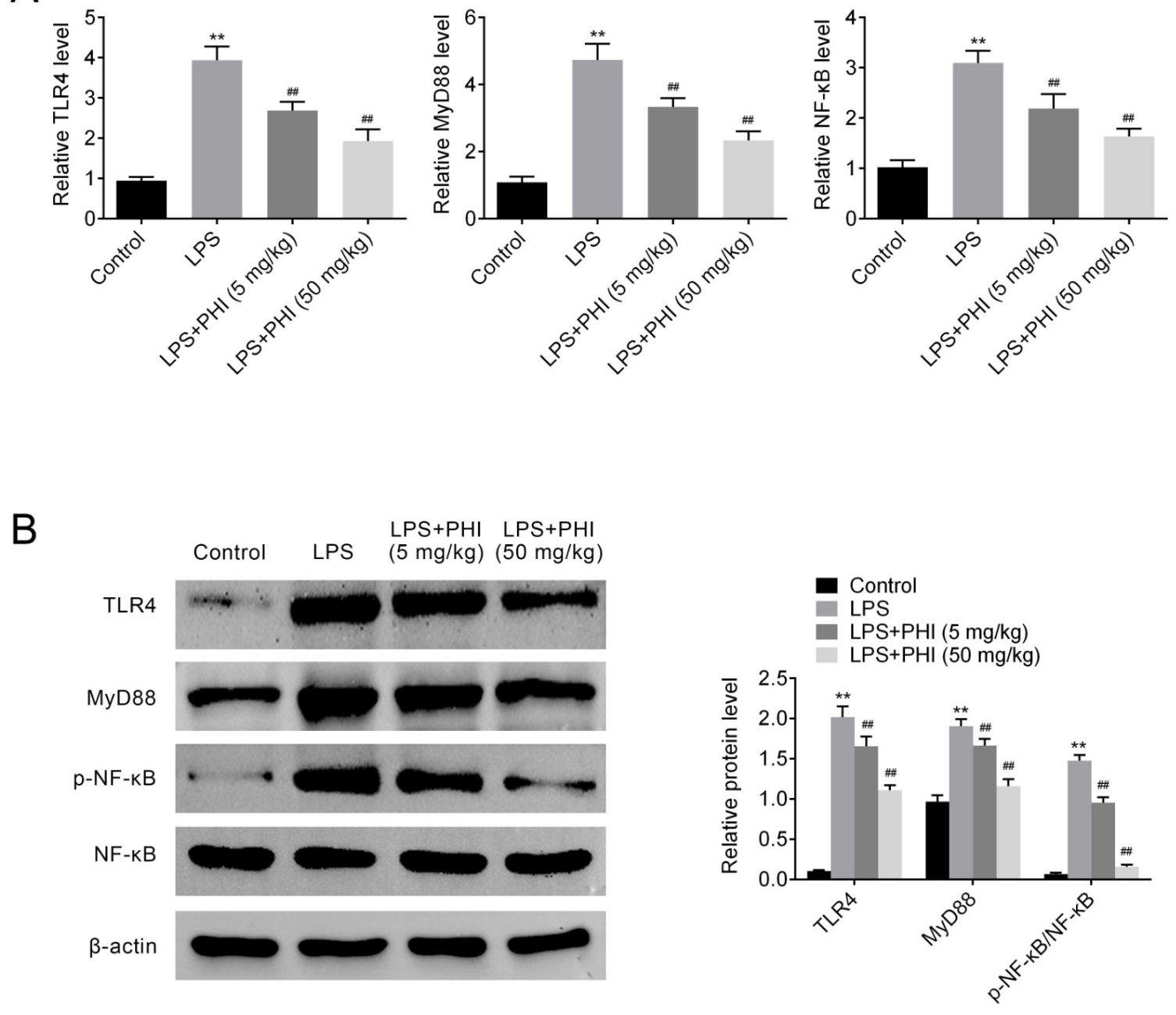

F I G U RE 4. PHI ameliorated LPS-induced inflammatory response by modulating the TLR4/MyD88/NF- $\kappa$ B pathway. (A) Mice were given different concentrations of PHI $(0,5$, and $50 \mathrm{mg} / \mathrm{kg})$ with an intragastric injection. (B) The expression of TLR4, MyD88, or NF- $\kappa \mathrm{B}$ in lung tissue. ${ }^{* *} p<0.01$ versus control group and ${ }^{\# \#} p<0.01$ versus LPS alone group.

LPS and plays a significant role in up-regulation and release of inflammatory cytokines in animal models of ALI [29]. LPS could trigger activation of TLR4, which in turn transmits signals by MyD88, resulting in the activation of NF- $\kappa \mathrm{B}$, which results in the upregulation of pro-inflammatory cytokines [30]. Notably, the PHI analog has also been reported to affect the expression level of TLR4 [31]. Therefore, it is vital to verify whether PHI ameliorates LPS-induced ALI by targeting TLR4. As expected, this study proved that PHI negatively regulated the expression of TLR4. In addition, western blot was further performed to assess the key protein expression levels in the TLR4/MyD88/NF- $\kappa$ B pathway. The results indicated that PHI downregulated the expression of MyD88 and NF- $\kappa \mathrm{B}$, which indicated that PHI reduced inflammatory response in LPSinduced ALI via modulating TLR4/MyD88/NF- $\kappa \mathrm{B}$ pathway.

\section{Conclusions}

In conclusion, this study proved that PHI ameliorated LPS-induced ALI by restraining inflammation via the TLR4/MyD88/NF- $\kappa$ B pathway. These findings shed light on the role of PHI in the pathogenesis of ALI and suggests a new potential therapeutic strategy against ALI.

\section{AUTHOR CONTRIBUTIONS}

MW and QC designed the study, supervised the data collection, analyzed the data, interpreted the data, prepare the manuscript for publication and reviewed the draft of the manuscript. All authors have read and approved the manuscript.

\section{ETHICS APPROVAL AND CONSENT TO PARTICIPATE}

Ethical approval was obtained from the Ethical Committee of the First Affiliated Hospital of Medical College of Shihezi University (Approval No. MCSU2019061).

\section{ACKNOWLEDGMENT}

Thanks to all the peer reviewers for their opinions and suggestions. 


\section{FUNDING}

This research received no external funding.

\section{CONFLICT OF INTEREST}

The authors state that there are no conflicts of interest to disclose.

\section{AVAILABILITY OF DATA AND MATERIALS}

All data generated or analyzed during this study are included in this published article.

\section{REFERENCES}

[1] de Luca D, Piastra M, Tosi F, Pulitano S, Mancino A, Genovese O, et al. Pharmacological therapies for pediatric and neonatal ALI/ARDS: an evidence-based review. Current Drug Targets. 2012; 13: 906-916.

[2] Bellani G, Laffey JG, Pham T, Fan E, Brochard L, Esteban A, et al. Epidemiology, patterns of care, and mortality for patients with acute respiratory distress syndrome in intensive care units in 50 countries. The Journal of the American Medical Association. 2016; 315: 788.

[3] Butt Y, Kurdowska A, Allen TC. Acute lung injury: a clinical and molecular review. Archives of Pathology \& Laboratory Medicine. 2016; 140: $345-350$.

[4] Mowery NT, Terzian WTH, Nelson AC. Acute lung injury. Current Problems in Surgery. 2020; 57: 100777.

[5] Mendez JL, Hubmayr RD. New insights into the pathology of acute respiratory failure. Current Opinion in Critical Care. 2005; 11: 29-36.

[6] Avlas O, Fallach R, Shainberg A, Porat E, Hochhauser E. Toll-like receptor 4 stimulation initiates an inflammatory response that decreases cardiomyocyte contractility. Antioxidants \& Redox Signaling. 2011; 15: 1895-1909.

[7] Liu L, Gu H, Liu H, Jiao Y, Li K, Zhao Y, et al. Protective effect of resveratrol against IL- $\beta$-induced inflammatory response on human osteoarthritic chondrocytes partly via the TLR4/MyD88/NF- $\kappa \mathrm{B}$ signaling pathway: an "in vitro study”. International Journal of Molecular Sciences. 2014; 15: 6925-6940.

[8] Hou Y, Luo S, Zhang Y, Jia Y, Li H, Xiao C, et al. Contrasting effects of acute and long-term corticosterone treatment on amyloid- $\beta$, betasecretase 1 expression, and nuclear factor kappa B nuclear translocation. Journal of Integrative Neuroscience. 2019; 18: 393-400.

[9] Castoldi A, Braga TT, Correa-Costa M, Aguiar CF, Bassi ÊJ, Correa-Silva $\mathrm{R}$, et al. TLR2, TLR4 and the MyD88 signaling pathway are crucial for neutrophil migration in acute kidney injury induced by sepsis. PLoS ONE. 2012; 7: e37584.

[10] Hung CH, Tsai Y, Li K. Phenolic antioxidants isolated from the flowers of Osmanthus fragrans. Molecules. 2012; 17: 10724-10737.

[11] Lee D, Lee S, Bang M, Park H, Lee T, Kim Y, et al. Lignans from the flowers of Osmanthus fragrans var. aurantiacus and their inhibition effect on no production. Archives of Pharmacal Research. 2011; 34: 20292035.

[12] Wang H, Cao Z-R. Anti-inflammatory effects of (-)-epicatechin in lipopolysaccharide-stimulated raw 264.7 macrophages. Tropical Journal of Pharmaceutical Research. 2014; 13: 1415.

[13] Hu N, Wang C, Dai X, Zhou M, Gong L, Yu L, et al. Phillygenin inhibits LPS-induced activation and inflammation of LX2 cells by

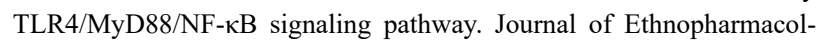
ogy. 2020; 248: 112361.

[14] National Research Council Committee for the Update of the Guide for the C, Use of Laboratory A. The national academies collection: reports funded by National Institutes of Health. Guide for the care and use of laboratory animals. Washington: National Academies Press. 2011.
[15] Ju M, Liu B, He H, Gu Z, Liu Y, Su Y, et al. MicroRNA-27a alleviates LPS-induced acute lung injury in mice via inhibiting inflammation and apoptosis through modulating TLR4/MyD88/NF- $\mathrm{BB}$ pathway. Cell Cycle. 2018; 17: 2001-2018.

[16] Wang N, Geng C, Sun H, Wang X, Li F, Liu X. Hesperetin ameliorates lipopolysaccharide-induced acute lung injury in mice through regulating the TLR4-MyD88-NF- $\kappa$ B signaling pathway. Archives of Pharmacal Research. 2019; 42: 1063-1070.

[17] Jiang Q, Yi M, Guo Q, Wang C, Wang H, Meng S, et al. Protective effects of polydatin on lipopolysaccharide-induced acute lung injury through TLR4-MyD88-NF- $\mathrm{B}$ pathway. International Immunopharmacology. 2015; 29: 370-376.

[18] Tianzhu Z, Shumin W. Esculin inhibits the inflammation of LPS-induced acute lung injury in mice via regulation of $\mathrm{TLR} / \mathrm{NF}-\kappa \mathrm{B}$ pathways. Inflammation. 2015; 38: 1529-1536.

[19] Tao W, Su Q, Wang H, Guo S, Chen Y, Duan J, et al. Platycodin D attenuates acute lung injury by suppressing apoptosis and inflammation in vivo and in vitro. International Immunopharmacology. 2016; 27: 138 147.

[20] Cheng N, Ren N, Gao H, Lei X, Zheng J, Cao W. Antioxidant and hepatoprotective effects of Schisandra chinensis pollen extract on CCl4induced acute liver damage in mice. Food and Chemical Toxicology. 2013; 55: 234-240.

[21] Kim SR, Lee MK, Koo KA, Kim SH, Sung SH, Lee NG, et al. Dibenzocyclooctadiene lignans from Schisandra chinensis protect primary cultures of rat cortical cells from glutamate-induced toxicity. Journal of Neuroscience Research. 2004; 76: 397-405.

[22] Sowndhararajan K, Deepa P, Kim M, Park SJ, Kim S. An overview of neuroprotective and cognitive enhancement properties of lignans from Schisandra chinensis. Biomedicine \& Pharmacotherapy. 2018; 97: 958968.

[23] Brandenberger C, Kling KM, Vital M, Christian M. The role of pulmonary and systemic immunosenescence in acute lung injury. Aging and Disease. 2018; 9: 553-565.

[24] Zhi H, Zhu H, Zhang Y, Lu Y, Li H, Chen D. In vivo effect of quantified flavonoids-enriched extract of Scutellaria baicalensis root on acute lung injury induced by influenza a virus. Phytomedicine. 2019; 57: 105-116.

[25] Luo Y, Pang X, Ansari AR, Wu X, Li H, Zhang Z, et al. Visfatin exerts immunotherapeutic effects in lipopolysaccharide-induced acute lung injury in murine model. Inflammation. 2020; 43: 109-122.

[26] Zhao G, Zhang T, Ma X, Jiang K, Wu H, Qiu C, et al. Oridonin attenuates the release of pro-inflammatory cytokines in lipopolysaccharide-induced RAW264.7 cells and acute lung injury. Oncotarget. 2017; 8: 6815368164.

[27] Chen C, Shi L, Li Y, Wang X, Yang S. Disease-specific dynamic biomarkers selected by integrating inflammatory mediators with clinical informatics in ARDS patients with severe pneumonia. Cell Biology and Toxicology. 2016; 32: 169-184.

[28] Alamgeer, Hasan UH, Uttra AM, Qasim S, Ikram J, Saleem M, et al. Phytochemicals targeting matrix metalloproteinases regulating tissue degradation in inflammation and rheumatoid arthritis. Phytomedicine. 2020; 66: 153134

[29] Sun H, Cai S, Zhang M, Zhao J, Wei S, Luo Y, et al. MicroRNA-206 regulates vascular smooth muscle cell phenotypic switch and vascular neointimal formation. Cell Biology International. 2017; 41: 739-748.

[30] Hoesel B, Schmid JA. The complexity of NF- $\kappa B$ signaling in inflammation and cancer. Molecular Cancer. 2013; 12: 86.

[31] Rahimifard M, Maqbool F, Moeini-Nodeh S, Niaz K, Abdollahi M, Braidy N, et al. Targeting the TLR4 signaling pathway by polyphenols: a novel therapeutic strategy for neuroinflammation. Ageing Research Reviews. 2017; 36: 11-19.

How to cite this article: Meiyan Wang, Qian Cai. Phillygenin attenuates LPS-induced acute lung injury of newborn mice in infantile pneumonia. Signa Vitae. 2021;17(4):171-177. doi:10.22514/sv.2021.085. 